\title{
Effect of a single session of aerobic walking exercise on arterial pressure in community-living elderly individuals
}

\author{
Leandra G Lima ${ }^{1}$, Júlio C Moriguti ${ }^{2}$, Eduardo Ferriolli ${ }^{2}$, Nereida KC Lima ${ }^{2}$
}

Several studies have demonstrated that one exercise session (ES) on a cycloergometer or ergometric treadmill causes a reduction in blood pressure (BP). However, there are few similar studies on walking, which is the exercise modality most available to the elderly. We investigated the immediate and 24-h effects of walking on BP in independent, community-living elderly individuals. Volunteers participated in a single ES and resting control session (CS). Before and after each session, BP was measured by auscultatory and oscillometric methods. After each session, 24-h ambulatory blood pressure monitoring was conducted. An accelerometer was installed $48 \mathrm{~h}$ before the sessions and left in place for 5 days. The mean volunteer age was $67.7 \pm 3.5$ years; 11 were hypertensive patients under treatment, and 12 were normotensive. In the total sample, there were immediate $14 \mathrm{~mm} \mathrm{Hg}$ and $12 \mathrm{~mm} \mathrm{Hg}$ reductions in systolic BP (SBP) after the ES according to the auscultatory and oscillometric methods, respectively. Diastolic BP (DBP) was reduced by $4 \mathrm{~mm} \mathrm{Hg}$ after the ES according to both methods. SBP during wakefulness and sleep and DBP during wakefulness were lower after the ES than after the CS $(P<0.01)$, when wakefulness and sleep were determined individually (variable-time pattern) using data from the activity monitors and provided by the volunteers. The variable-time pattern was more effective in detecting reductions in BP than the fixed-time pattern. Hypertension Research (2012) 35, 457-462; doi:10.1038/hr.2011.227; published online 9 February 2012

Keywords: actigraph; aerobic exercise; ambulatory blood pressure monitoring; blood pressure; elderly individuals

\section{INTRODUCTION}

The aging process is associated with many important physiological changes, of which increased blood pressure (BP) is one of the most well known. In addition to regular physical exercise, a single session of acute exercise has also been observed to reduce BP during the recovery period.

Hypotensive responses have been observed in hypertensive animals ${ }^{1,2}$ and normotensive (NT) and hypertensive humans. ${ }^{3-6}$ The magnitude of the hypotensive response to aerobic exercise varies between NT and hypertensive subjects and young and elderly subjects. In a study conducted in NT and hypertensive elderly persons, ${ }^{6}$ reduced BP was observed only in hypertensive subjects after executing a single exercise session (ES) on a cycloergometer. In another study including only NT women, ${ }^{7}$ a marked reduction in BP was observed after acute dynamic exercise in middle-aged and elderly women but not in young women. Other studies ${ }^{8,9}$ have shown greater BP reductions in persons aged 41-60 years compared with older and younger individuals.

Ambulatory arterial blood pressure monitoring (ABPM) has been extensively used to obtain more detailed information about BP oscillations because it permits measurement during routine activities and sleep. Strict definitions of sleep and wakefulness periods have been used for many years to evaluate the measurements obtained by ABPM. However, these definitions may not correspond with real sleep patterns and may therefore lead to interpretation errors. ${ }^{10}$ For this reason, accelerometry-based actigraphs are used as an auxiliary tool for investigating variations in sleep patterns, behavioral effects and treatment interventions. ${ }^{11}$

Although the literature shows reduced BP after both acute and regular physical exercise, to the best of our knowledge this has not been investigated using walking on a track with older individuals. Because the main form of exercise available for the elderly is walking, this study aimed to evaluate the hemodynamic responses of older volunteers after a single walking session of moderate intensity and duration, and the effect of the ES on the intensity of their usual activities. Our hypotheses were that this type of exercise would be effective for reducing BP and that the use of an accelerometry-based actigraph would improve the quality of data obtained during wakefulness and sleep periods.

\footnotetext{
${ }^{1}$ Department of Internal Medicine, School of Medicine of Ribeirão Preto, University of São Paulo, Ribeirão Preto, Brazil; ${ }^{2}$ Department of Internal Medicine, Division of General Clinical Medicine and Geriatrics, School of Medicine of Ribeirão Preto, University of São Paulo, Ribeirão Preto, Brazil

Correspondence: Professor NKC Lima, Department of Internal Medicine, Division of General Clinical Medicine and Geriatrics, School of Medicine of Ribeirão Preto, University of São Paulo, Av. Bandeirantes, 3900, Ribeirão Preto, SP 14048-900, Brazil.

E-mail: nereida@fmrp.usp.br

Received 16 May 2011; revised 10 October 2011; accepted 11 October 2011; published online 9 February 2012
} 


\section{METHODS}

One hundred elderly volunteers (60 years of age and older) were randomly selected (one for every 20 names) from approximately 2000 individuals born between 1929 and 1944 registered in the Family Health Centers linked to the School of Medicine of Ribeirão Preto. We aimed for a sample size of 52 volunteers (power of $80 \%$, considering a s.d. of $9 \mathrm{~mm} \mathrm{Hg}$ and a difference of $5 \mathrm{~mm} \mathrm{Hg}$ between resting and exercise states). From the 100 volunteers initially selected, 53 were excluded according to the following criteria: positive treadmill test for ischemia (5); use of beta-blockers (17); consumption of more than seven alcoholic beverages per week or smoking (15); hemoglobin values below $12 \mathrm{~g} \mathrm{ml}^{-1}$, presence of arrhythmias; previous diagnosis of cerebrovascular, renal, pulmonary and musculoskeletal diseases or diabetes (16); hypothyroidism or hyperthyroidism; individuals with more than a $10 \mathrm{~mm} \mathrm{Hg}$ difference between the mean BP obtained from the two upper limbs; and individuals unable to keep a diary of activities (illiterate subjects with no family support). During the study, 18 volunteers withdrew consent and 6 volunteers were excluded because they had systolic BP (SBP) $\geqslant 160$ and/or diastolic BP (DBP) $\geqslant 100 \mathrm{~mm} \mathrm{Hg}$ during two different assessments, demonstrating greater risk in performing the ESs. Therefore, 23 volunteers completed the study.

The study was approved by the Research Ethics Committee of the Teaching Health Center at the School of Medicine of Ribeirão Preto (0169/CEP/CESFMRP-USP), and all volunteers provided written informed consent prior to participation.

BP was measured six times on each arm: three times using the auscultatory method (mercury sphygmomanometer, MISSOURI, Embu, Brazil) and three times using the oscillometric method (OMRON, Shanghai, China, HME-431) at 5-min intervals. On the second consecutive day, BP was measured again in the same manner on only the right arm. The volunteers were considered to be hypertensive when the mean BP value obtained by the auscultatory method was $140 \times 90 \mathrm{~mm} \mathrm{Hg}$ or higher ${ }^{12}$ or if they were already taking antihypertensive medication.

The maximum aerobic capacity was assessed by applying a maximum treadmill test (Inbrasport Master I, IBRAMED, Sao Paulo, Brazil) using the Bruce protocol, with a velocity between 1.7 and $5 \mathrm{~km} \mathrm{~h}^{-1}$. The test was interrupted by the observer when the volunteer achieved maximum performance, reaching the maximum predicted heart rate (HR), ${ }^{13}$ or when the observer felt it was necessary to discontinue the test due to electrocardiographic changes or other abnormalities. Those who did not reach the maximum HR were excluded from the protocol.

Volunteers were classified as active or sedentary based on the maximum oxygen consumption obtained by the American College of Sports Medicine formula ${ }^{13}$ and adapted conditioning tables ${ }^{14} \quad\left(\mathrm{VO}_{2} \quad \max =\{[(\right.$ maximum speed $\times 16.66) \times 0.1]+3.5\}+\{[($ maximum slope $/ 100) \times($ maximum speed $\times 16.66)]$ $\times 1.8\}$ ).

After the subjects were classified in terms of physical conditioning, their working $\mathrm{HR}$ was determined by the following formula: $\mathrm{HRT}=$ (maximum HRresting HR $) \times \_\%+$ resting HR. ${ }^{12}$ Maximum HR was established by the maximum ergometric test, and resting HR was measured before the test. Two percentages were used for the lower and upper limits of the training range: $50 \%$ and $70 \%$ for the sedentary volunteers and $60 \%$ and $80 \%$ for conditioned volunteers, respectively. ${ }^{12}$ We opted for different percentages for sedentary and active volunteers to avoid under- and overestimating physical ability according to the guidelines for prescribing aerobic exercise. ${ }^{12}$ Subjects were asked not to participate in other exercise programs, to maintain regular use of their medications and to continue their daily routine during the study.

The actigraph (Actiwatch Mini Mitter Company, Sunriver, OR, USA) was installed 1 week after the treadmill test. The monitor was placed on the nondominant wrist, and activity (counts) was continuously recorded at 1-min intervals during wakefulness and sleep.

After the evaluations described above, the volunteers were assigned to begin the exercise or resting protocols according to randomization using the SAS system, version 9 (SAS Institute, Cary, NC, USA).

The sessions were held at 1-week intervals, and both sessions were performed from 0700 to 0900 hrs. Volunteers were instructed to take their regular medications and eat a light breakfast (the amount subjects were previously accustomed to) at 0600 hrs. Initially, BP was measured every 5 min for a period of $20 \mathrm{~min}$ in the seated position, with the auscultatory device installed on the right arm and the oscillometric device on the left arm. The two devices were insufflated simultaneously to measure pulse rate (PR) (Polar S610, POLAR, Warwick, UK). PR was used to estimate HR.

The ES was performed by walking on a track, and the session began with a 5min warm-up during which volunteers were asked to walk at their usual speed. After the warm-up, the walking speed was increased to ensure that each subject maintained his or her individually prescribed HR for $30 \mathrm{~min}$. PR and the perception of physical effort (Borg Scale) ${ }^{15}$ were recorded every $10 \mathrm{~min}$. Slowdown $(5 \mathrm{~min})$ was then initiated, during which volunteers were instructed to reduce their walking intensity. After stopping, volunteers were asked to rest for $40 \mathrm{~min}$ in a seated position.

At the end of the sessions, the devices for BP measurement were re-installed, and BP and PR were measured every $5 \mathrm{~min}$ for an additional $60 \mathrm{~min}$, resulting in 12 total measurements. All BP measurements were performed by the same trained investigator. After the measurements, at about $0930 \mathrm{hrs}$, the ABPM monitor (Spacelabs 90207, Spacelabs Healthcare, Issaquah, WA, USA) was installed on the non-dominant arm and left in place for a continuous 24-h period. The 24-h period was divided into probable wakefulness and sleep periods. Wakefulness was predetermined as the period from 0700 to $2300 \mathrm{hrs}$, and sleep was defined as the period from 2300 to $0700 \mathrm{hrs}$, with the device programmed for measurements at 15-min intervals during wakefulness and 20min intervals during sleep. This standard criterion for determining wakefulness and sleep was called the 'fixed-time pattern' in the present study. Wakefulness and sleep were also determined individually for the data analysis based on information reported by the patients (times when they went to bed and when they awoke) together with the times calculated by the activity-monitoring software. This evaluation method was called the 'variable-time pattern' in the present study.

\section{Statistical analysis}

Data were reported as mean \pm s.d., and BP (auscultatory and oscillometric) and ABPM were statistically analyzed using the linear fixed-effects model, including interactions between periods (pre- and post-sessions) for BP and between activities (exercise and rest) for BP and ABPM. This model was adjusted for gender, randomization and physical conditioning levels. A twotailed Student's $t$-test was used to compare the spontaneous activity measured by the activity monitor. Differences were considered statistically significant when $P<0.05$. The SAS system (version 9; SAS Institute) was used for all statistical calculations.

\section{RESULTS}

Twelve of the elderly volunteers were classified as NT and 11 as hypertensive (HT). Sixteen individuals were women. The anthropometric and cardiovascular characteristics of the total sample (TS) and both groups are presented in Table 1 . Nine HT patients were regularly taking diuretics, calcium channel blockers (dihydropyridine) or angiotensin enzyme inhibitors. All volunteers reached the maximum HR during the treadmill test. The mean maximum HR reached in the test was $153 \pm 11$ beats per min, and the peak oxygen consumption was $27.5 \pm 8 \mathrm{ml} \mathrm{kg}^{-1} \mathrm{~min}$. The maximum energy expenditure was 7.8 MET. Based on the maximum $\mathrm{VO}_{2}$ values obtained, the HT group was composed of four sedentary and seven active elderly volunteers, and the NT group was composed of three sedentary and eight active volunteers.

The mean total spontaneous activity recorded by the activity monitor during the 2 days preceding the rest and ESs did not differ between the pre-rest and pre-exercise periods, and no differences in pressure levels or PR were observed during the pre-intervention period for the exercise and rest sessions, suggesting that SBP, DBP and PR values were similar before sessions in the TS and HT and NT groups $(P>0.05)$.

All volunteers were able to complete the 40-min sessions. PR significantly increased at the end of the ES compared with the end 
Table 1 Anthropometric and clinical characteristics of the elderly subjects as a whole and of the normotensive and hypertensive groups

\begin{tabular}{|c|c|c|c|c|}
\hline Characteristics & Total sample & Normotensive group & Hypertensive group & $P^{*}$ \\
\hline Age (years) & $67.7 \pm 3.4$ & $67.1 \pm 2.8$ & $68.3 \pm 4.0$ & $>0.05$ \\
\hline Gender (male/female) & $7 / 16$ & $5 / 7$ & $2 / 9$ & $>0.05$ \\
\hline Weight (kg) & $70.4 \pm 10.0$ & $70.8 \pm 11.2$ & $70.0 \pm 8.9$ & $>0.05$ \\
\hline Height (m) & $1.58 \pm 0.10$ & $1.60 \pm 0.10$ & $1.56 \pm 0.10$ & $>0.05$ \\
\hline BMI $\left(\mathrm{kg} / \mathrm{m}^{2}\right)$ & $28.1 \pm 4.0$ & $27.8 \pm 4.0$ & $28.3 \pm 4.0$ & $>0.05$ \\
\hline DBP sitting (mm Hg) RA, OSC. & $71 \pm 10$ & $70 \pm 6$ & $73 \pm 10$ & $>0.05$ \\
\hline DBP sitting $(\mathrm{mm} \mathrm{Hg}) \mathrm{RA}$, AUS. & $72 \pm 10$ & $70 \pm 4$ & $75 \pm 12$ & $>0.05$ \\
\hline Resting HR & $73 \pm 8$ & $72 \pm 9$ & $74 \pm 6$ & $>0.05$ \\
\hline HTR, lower limit & $119 \pm 8$ & $119 \pm 8$ & $118 \pm 7$ & $>0.05$ \\
\hline HTR, upper limit & $135 \pm 10$ & $136 \pm 10$ & $134 \pm 9$ & $>0.05$ \\
\hline
\end{tabular}

Abbreviations: AUS, auscultatory (mercury column); BMI, body mass index (weight/height ${ }^{2}$ ); DBP, diastolic blood pressure; HR, heart rate; HTR, training heart rate; OSC, oscillometric (OMRON); $\mathrm{RA}$, right arm; SBP, systolic blood pressure.

Data are reported as mean \pm s.d.

$P^{\star}$ : Normotensive vs. Hypertensive group.

of the rest session $(P<0.01)$ and pre-exercise period $(P<0.01)$. During the rest session, NT volunteers had lower PR values than HT volunteers $(P<0.05)$.

Compared with the pre-exercise values, SBP and DBP were reduced by $14 \mathrm{~mm} \mathrm{Hg}$ and $4 \mathrm{~mm} \mathrm{Hg}$, respectively, in the TS during the first hour after exercise using the auscultatory method and $12 \mathrm{~mm} \mathrm{Hg}$ and $4 \mathrm{~mm} \mathrm{Hg}$, respectively, using the oscillometric method $(P<0.01)$. In the HT group, $18 \mathrm{~mm} \mathrm{Hg}$ and $17 \mathrm{~mm} \mathrm{Hg}$ reductions were observed in auscultatory and oscillometric SBP, respectively $(P<0.01)$, and reductions of $4 \mathrm{~mm} \mathrm{Hg}(P=0.02)$ and $6 \mathrm{~mm} \mathrm{Hg}(P<0.01)$ were observed in auscultatory and oscillometric DBP, respectively. In the NT group, $10 \mathrm{~mm} \mathrm{Hg} \quad(P<0.01)$ and $8 \mathrm{mmHg} \quad(P<0.01)$ reductions were observed in auscultatory and oscillometric SBP, respectively, and reductions of $3 \mathrm{~mm} \mathrm{Hg}(P=0.02)$ and $2 \mathrm{mmHg}(P=0.06)$ were observed in auscultatory and oscillometric DBP, respectively, as illustrated in Figures 1 and 2.

$\mathrm{BP}$ was reduced for $24 \mathrm{~h}$ following acute exercise compared with rest in the TS, with a $3 \mathrm{~mm} \mathrm{Hg}$ reduction in SBP $(95 \% \mathrm{CI}(1.82 ; 3.27))$ and a $2 \mathrm{~mm} \mathrm{Hg}$ reduction in DBP $(1.53 ; 2.77)(P<0.01)$. Similar reductions were observed during wakefulness and sleep, as shown in Table 2.

In the HT group, BP reductions were also observed following acute exercise compared with rest, with $3 \mathrm{~mm} \mathrm{Hg}(P<0.01)$ reductions in SBP and $4 \mathrm{~mm} \mathrm{Hg}(P<0.01)$ reductions in DBP, whereas in the NT group, $2 \mathrm{~mm} \mathrm{Hg}(P<0.01)$ and $1 \mathrm{~mm} \mathrm{Hg}(P=0.02)$ reductions were observed for SBP and DBP, respectively. Post-exercise BP was lower during both wakefulness and sleep compared with post-rest $(P<0.01)$, except for DBP during sleep in the NT group, which remained unchanged.

In the point-by-point (hourly means) ABPM BP analyses after the ES, significant reductions in SBP compared with the rest session were observed in the TS at $1,2,3,10,11,12$ and $18 \mathrm{~h}$ after device placement. Significant reductions in DBP compared with the rest session were observed at $2,3,7,10,12,13,18$ and $20 \mathrm{~h}$, as illustrated in Figure 3. For the NT and HT groups, significant reductions in SBP occurred at 2,14 and $18 \mathrm{~h}$ and $3,5,6,7,10,11,12,13,14,15$ and $18 \mathrm{~h}$ after device placement, respectively. Significant reductions in DBP occurred at 6,12 and $20 \mathrm{~h}$ and $2,3,7,10,12,13,14,15$ and $18 \mathrm{~h}$ in the NT and HT groups, respectively.

In the present study, the variable-time pattern (activity monitor) was more effective in detecting reductions in BP during sleep and
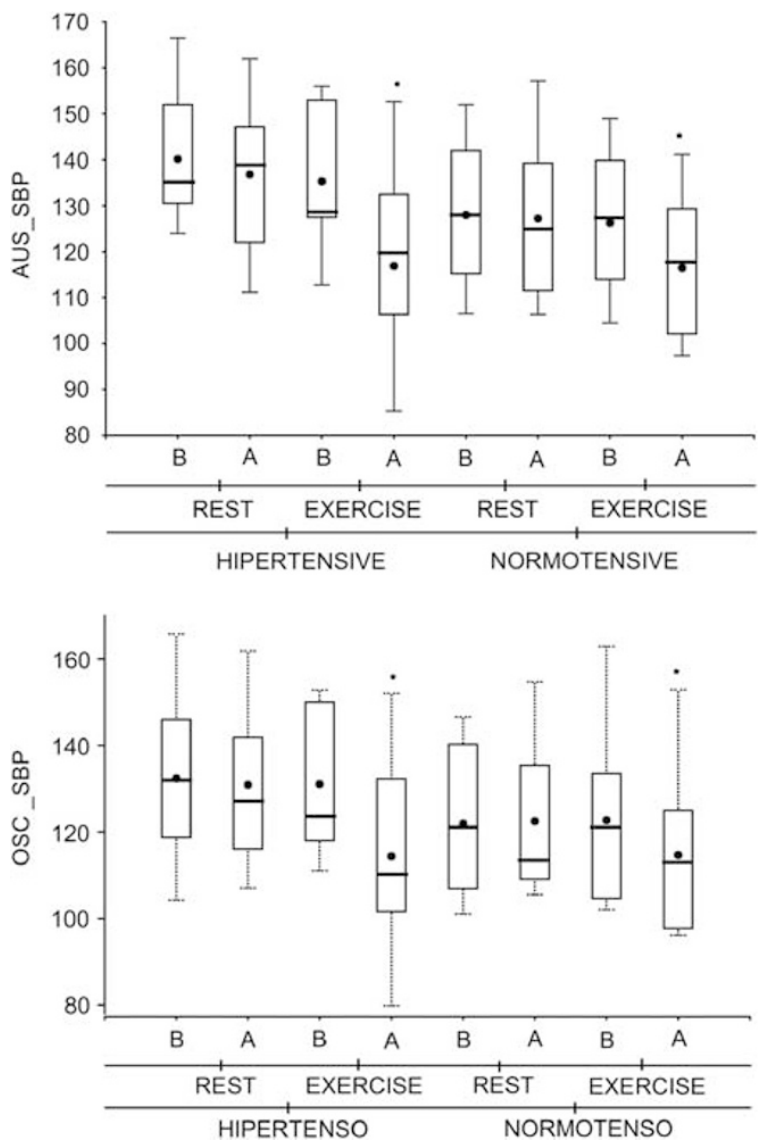

Figure 1 Systolic blood pressure (SBP) measured with the auscultatory (AUS) and oscillometric (OSC) methods pre- (B) and post- (A) exercise and rest sessions in hypertensive (HT) and normotensive (NT) volunteers. HT Group: ${ }^{*} P<0.01$ vs. post-rest; $P<0.01$ vs. pre-exercise. NT Group: ${ }^{\star} P<0.01$ (AUS) and $P=0.02$ (OSC) vs. post-rest; $P<0.01$ vs. pre-exercise.

wakefulness after exercise than the fixed-time pattern, as shown in Table 3.

When the pre-exercise period was compared with the post-exercise period, no significant differences were detected for total activity 

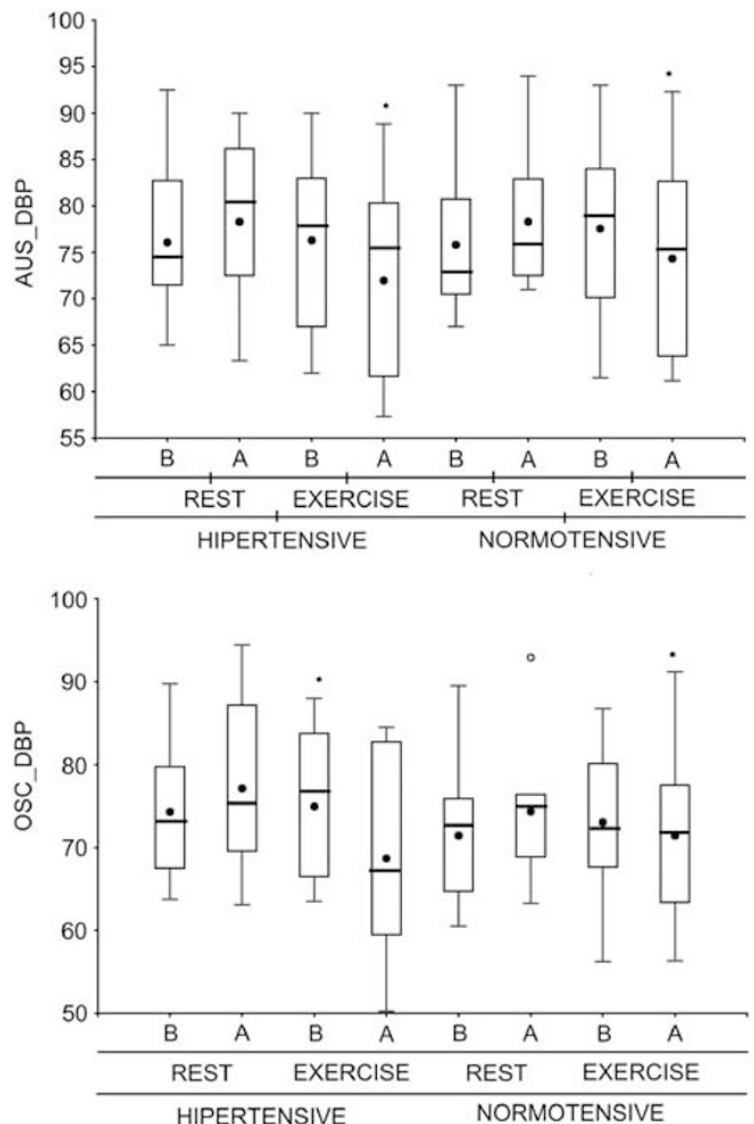

Figure 2 Diastolic blood pressure (DBP) measured with the auscultatory (AUS) and oscillometric (OSC) methods pre- (B) and post- (A) exercise and rest sessions in hypertensive (HT) and normotensive (NT) volunteers. HT Group: ${ }^{*} P<0.01$ vs. post-rest; ${ }^{*} P=0.02$ (AUS) and $P<0.01$ (OSC) vs. preexercise. (NT Group): ${ }^{*} P=0.02$ (AUS) vs. post-rest; $P=0.06$ (AUS) vs. preexercise.

Table 2 Comparison of the differences in blood pressure between the exercise and rest sessions during sleep and wakefulness

\begin{tabular}{llllllll} 
Period & Variable & Session & Session & Diff. & $95 \% \mathrm{Cl}$ & $95 \% \mathrm{ClS}$ & P-value \\
\hline \multirow{2}{*}{ Sleep } & Systolic & Rest & Exercise & 2.51 & 1.09 & 3.94 & $<0.01$ \\
& Diastolic & Rest & Exercise & 2.17 & 0.95 & 3.38 & $<0.01$ \\
\multirow{4}{*}{ Wakefulness } & Systolic & Rest & Exercise & 2.93 & 2.16 & 3.70 & $<0.01$ \\
& Diastolic & Rest & Exercise & 2.27 & 1.62 & 2.93 & $<0.01$ \\
\hline
\end{tabular}

Abbreviations: $\mathrm{Cl}$, confidence interval inferior, $\mathrm{CIS}$, confidence interval superior.

(activity monitor) in the TS (134 102 vs. 101886 counts, $P=0.10)$ or NT (123475 vs. 86652 counts, $P=0.06)$ or HT (145695 vs. 118504 counts, $P=0.65)$ groups.

\section{DISCUSSION}

Few studies have evaluated the effect of acute exercise on the elderly population, and to the best of our knowledge there have been no investigations of aerobic exercise performed on a track using walking as the exercise modality, including evaluations of habitual physical activity and BP in an acute exercise protocol. Previous studies have been performed on treadmills or cycloergometers.
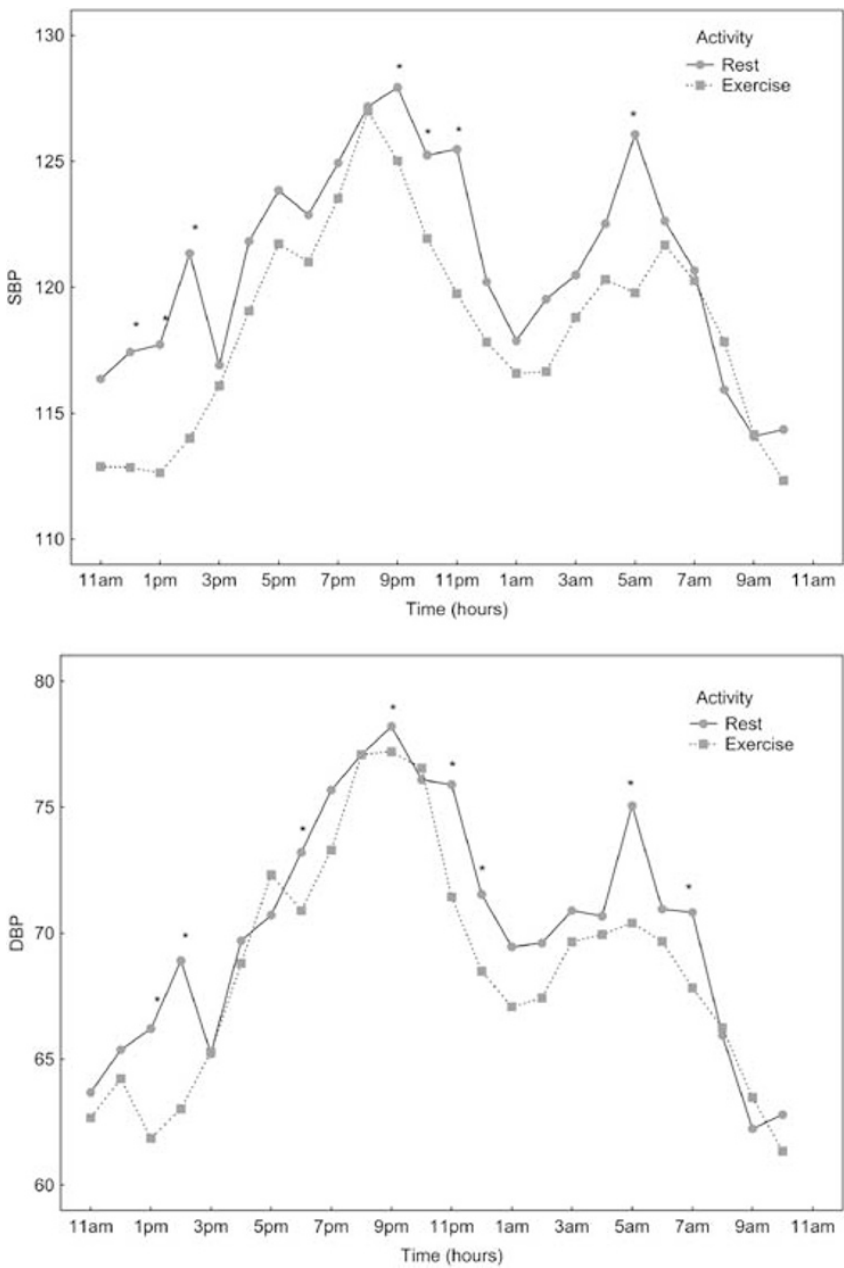

Figure 3 Systolic blood pressure (SBP) and diastolic blood pressure (DBP) over a 24-h period post-exercise and rest sessions (general $P<0.01$ ). ${ }^{*} P<0.05$. A full color version of this figure is available at the Hypertension Research journal online.

In the present study, less than half of the 100 elderly individuals initially selected were included in the physical activity protocol because the selection criteria were rigorous. We excluded volunteers with diseases or factors commonly occurring in the elderly population, such as diabetes (for example, in Brazil, the prevalence of diabetes is high, with $17.3 \%$ of persons aged 60-69 years being affected), ${ }^{16}$ smoking (the prevalence of tobacco use among the elderly is $18.8 \%$ for both genders) ${ }^{17}$ and the use of beta-blockers. We understand that these criteria do not prevent the execution of acute exercise, but it would be difficult to evaluate hemodynamic effects in such varying subgroups. In addition, $16 \%$ of the elderly subjects did not agree to participate in the study.

In this study, post-exercise SBP was significantly reduced during the first $60 \mathrm{~min}$ compared with pre-exercise and post-rest values. The same results were observed ${ }^{18}$ in volunteers trained on a cycloergometer at $70 \%$ of peak $\mathrm{VO}_{2}$; $\mathrm{SBP}$ was lower during the first hour after exercise than pre-exercise.

DBP was less responsive than SBP, although it was also significantly reduced. Several investigators have also detected greater reductions in $\mathrm{SBP}^{19,20,21}$ than in DBP. When the TS was subdivided into NT and HT groups, greater reductions in BP during the first hour post-exercise were observed in the HT group than in the NT 
Table 3 Comparison of mean blood pressure between the exercise and rest sessions in each method

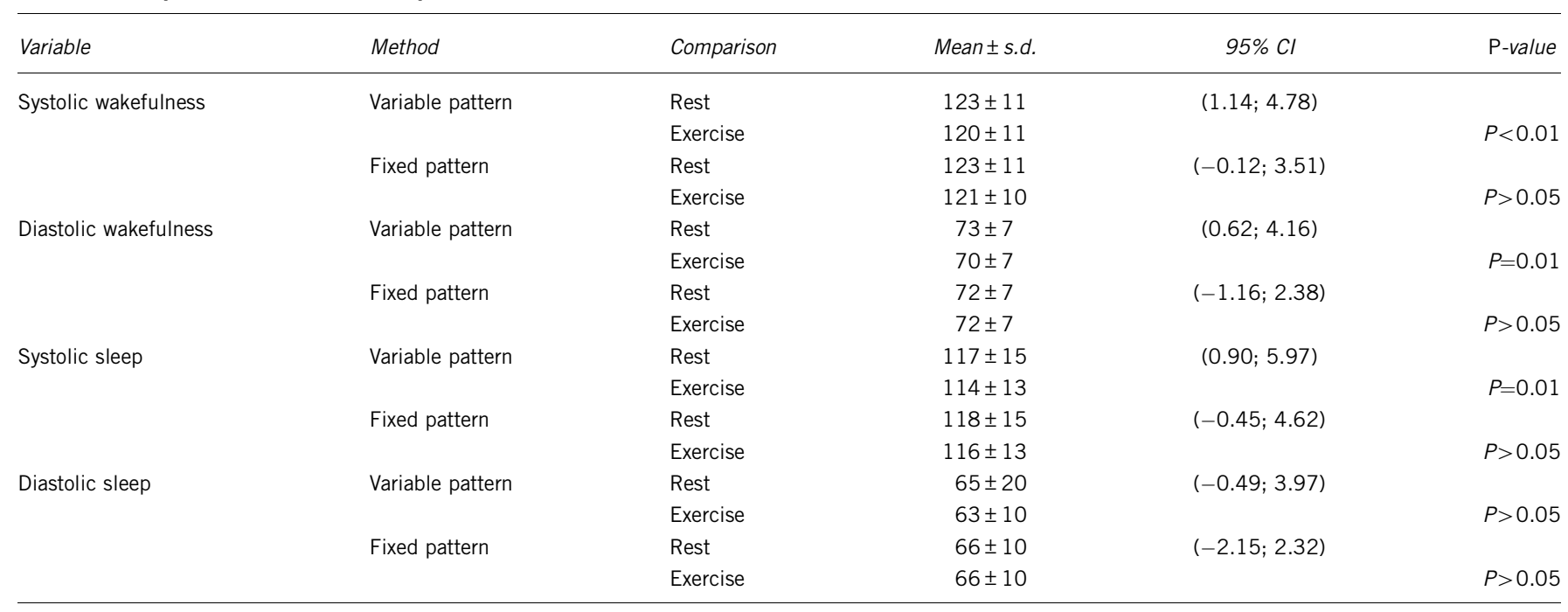

Abbreviation: $\mathrm{Cl}$, confidence interval.

group, although the reduction was still significant in the latter group. Many investigators ${ }^{6,22-24}$ have observed post-exercise reductions in BP only in hypertensive persons, regardless of age. The results reported by these investigators have suggested that acute physical exercise does not promote BP reductions in NT individuals. However, other studies have detected post-exercise BP reductions in this subgroup. ${ }^{3,5,25,26}$ Consistent with the present study, other investigators have reported that $\mathrm{BP}$ reductions are greater in hypertensive than in NT persons. ${ }^{27,28}$

Regarding the effect of exercise duration and intensity on BP reduction, the present results were similar to those reported in other studies conducted with elderly individuals. ${ }^{4}$ Elderly individuals submitted to 40 min of exercise at $50 \%$ of maximum $\mathrm{VO}_{2}$ (calculated in an indirect manner) presented significant reductions of $10 \mathrm{~mm} \mathrm{Hg}$ and $6 \mathrm{~mm} \mathrm{Hg}$ for women and $10 \mathrm{~mm} \mathrm{Hg}$ and $5 \mathrm{~mm} \mathrm{Hg}$ for men in SBP and DBP, respectively. Brownley et al. ${ }^{29}$ observed significant reductions in SBP for up to $5 \mathrm{~h}$, after $20 \mathrm{~min}$ of exercise on a cycloergometer at $60-70 \%$ of the predicted HR for age. According to Hamer et al., ${ }^{30}$ the ideal exercise duration and intensity required to obtain lower BP and maintain NT conditions have not been fully clarified.

In the present study, basal PR values did not differ between the preexercise and pre-rest values. Post-exercise PR was elevated for the first hour, in contrast to post-rest PR, which was significantly reduced in the TS and NT and HT groups. Transitory tachycardia after a prolonged ES has also been observed in other studies, ${ }^{6,31,32}$ which have reported significantly increased PR and decreased BP in hypertensive elderly persons during the post-exercise period. Other investigators observed the same simultaneous $\mathrm{HR}$ elevations and BP reductions in young and adult subjects. ${ }^{23,25,27}$

Another relevant result of the present investigation was that SBP was reduced for $24 \mathrm{~h}$. This result differs from those reported by other investigators $^{32,33}$ who observed only short-term reductions in BP, but it agrees with other authors $6,22,34$ who observed prolonged BP reductions.

Both sleep and wakefulness data were analyzed in this study. This variable-time pattern, using data from the activity monitors and data provided by the volunteers regarding sleeping time, was more effective in detecting reduced BP than a fixed-time pattern.

Peixoto-Filho et al. ${ }^{36}$ compared the results with the arbitrary (fixed) pattern of patient-reported wakefulness and sleep times in regard to mean wakefulness BP, sleep BP and wakefulness-sleep BP differences in 50 subjects and found that the differences were clinically relevant in as many as $30 \%$ of patients. Gosse et al. ${ }^{37}$ also showed that a significant proportion of subjects classified as NT when assessed during the daytime were classified as hypertensive when the values were obtained during both wakefulness and sleep. However, there is still no consensus as to how these variables interact with hypertension classifications according to the Guidelines of the European Society of Hypertension. ${ }^{3,5}$ We emphasize the importance of an activity diary as a complementary method for subjectively determining sleep periods, which is essential for documenting the events occurring during the activity-recording period.

In this study, no differences were detected in spontaneous physical activity pre- and post-ESs. Reductions in spontaneous activity due to asthenia have been reported to occur after acute ESs, especially after the resistance to activity in untrained volunteers, which may indicate the need for caution when prescribing this type of activity to elderly subjects. ${ }^{38,39}$

In conclusion, a single session of aerobic exercise with walking was effective for immediate and late BP reductions in elderly volunteers. The use of activity monitors to determine the actual periods of sleep was more effective for detecting reductions in BP during sleep than the fixed-time method. A single ES did not interfere with the habitual spontaneous activity of volunteers.

\section{ACKNOWLEDGEMENTS}

We wish to thank Iara Felício Anunciato and Fernanda Jatte (post-graduate students at the School of Medicine at Ribeirão Preto), Paulo Padovan (physical education instructor), and the Coca-Cola Foundation-Pemberton Prize for stimulating research in Brazil (2009). This research was supported by the National Council for Scientific and Technological Development (CNPq) and Foundation for the Support of Teaching, Research and Care - Clinics Hospital, Faculty of Medicine of Ribeirao Preto, University of Sao Paulo. 
1 Véras-Silva AS, Mattos KC, Gava NS, Brum PC, Negrão CE, Krieger EM. Low-intensity exercise training decreases cardiac output and hypertension in spontaneously hypertensive rats. Am J Physiol 1997; 273: H2627-H2631.

2 Lizardo JH, Silveira EA, Vassallo DV, Oliveira EM. Post-resistance exercise hypotension in spontaneously hypertensive rats is mediated by nitric oxide. Clin Exp Pharmacol Physiol 2008; 35: 782-787.

3 Jones H, George K, Edwards B, Atkinson G. Is the magnitude of acute post-exercise hypotension mediated by exercise intensity or total work done? Eur J Appl Physiol 2007; 102: 33-40.

4 Kario K, Schwartz JE, Pickering TG. Ambulatory physical activity as a determinant of diurnal blood pressure variation. Hypertension 1999; 34: 685-691.

5 Forjaz CLM, Santaella DF, Rezende LO, Barretto ACPB, Negrão CE. Exercise duration determines and duration of post-exercise hypotension. Arq Bras Cardiol 1998; 70: 99-104.

6 Rondon MUP, Alves MJNN, Braga AMFW, Texeira OTUN, Barreto ACP, Krieger EM, Negrão CE. Postexercise blood pressure reduction in elderly hypertensive patients. J Am Coll Cardiol 2002; 39: 676-682.

7 Harvey PJ, Morris BL, Kubo T, Picton PE, Su WS, Notarius CF, Floras JS. Hemodynamic after-effects of acute dynamic exercise in sedentary normotensive postmenopausal women. J Hypertens 2005; 23: 285-292.

8 Ivy JL, Goforth Jr HW, Damon BM, McCauley TR, Parsons EC, Price TB. Early postexercise muscle glycogen recovery is enhanced with a carbohydrate-protein supplement. J App/ Physiol 2002; 93: 1337-1344.

9 Suzuki S, Ohta T. Exercise therapy for patients with mild hypertension. Nippon Rinsho 2008; 66: 1553-1559.

10 Gatzka CD, Schmieder RE. Improved classification of dippers by individualized analysis of ambulatory blood pressure profiles. Am J Hypertens 1995; 8: 666-671.

11 Acebo C, LeBourgeois MK. Actigraphy. Respir Care Clin N Am 2006; 121 : 23-30.

12 Sociedade Brasileira de Cardiologia - SBC, Sociedade Brasileira de Hipertensão - SBH, Sociedade Brasileira de Nefrologia - SBN. V Brazilian Guidelines in Arterial Hypertension. Arq Bras Cardiol 2007; 89: e24-e79.

13 American College of Sports Medicine. ACSM's Guidelines for Exercise Testing and prescription. Guanabara Koogan: Rio de Janeiro, 2003 p. 239.

14 Powers SK, Howley ET. Exercise Physiology: Theory and Application to Fitness and Performance, Ed.: 2000, Cap.15. Manole, Säo Paulo, Brazil, 2000 p. 270.

15 Borg CAV. Psychophysical bases of perceived exertion. Med Sci Sports Exerc 1982; 14 377-381.

16 Viegas-Pereira APF, Rodrigues RN, Machado CJ. Factors associated with the prevalence of diabetes as self-reported by elderly persons in the State of Minas Gerais, Brazil, 2003. Rev Bras Estu Popul 2008; 25: 365-376.

17 Marinho V, Blay SL, Andreoli SB, Gastal FL. A prevalence study of current tobacco smoking in later life community and its association with sociodemographic factors, physical health and mental health status. Soc Psychiatry Psychiatr Epidemiol 2008; 43: 490-497.

18 MacDonald JR. Potential causes, mechanisms, and implications of post exercise hypotension. J Hum Hypertens 2002; 16: 225-236.

19 Padilla J, Wallace JP, Park S. Accumulation of physical activity reduces blood pressure in pre- and hypertension. Med Sci Sports Exerc 2005; 37: 1264-1275.

20 Park S, Rink L, Wallace J. Accumulation of physical activity: blood pressure reduction between 10-min walking sessions. J Hum Hypertens 2008; 22: 475-482.
21 Jones H, Atkinson G, Leary A, George K, Murphy M, Waterhouse J. Reactivity of ambulatory blood pressure to physical activity varies with time of day. Hypertension 2006; 47: 778-784.

22 Quinn TJ. Twenty-four hour, ambulatory blood pressure responses following acute exercise: impact of exercise intensity. J Hum Hypertens 2000; 14: 547-553.

23 Pescatello LS, Fargo AE, Leach CN, Scherzer HH. Short term effect of dynamic exercise on blood pressure. Circulation 1991; 83: 1557-1561.

24 Fernandez S, Scales KL, Pineiro JM, Schoenthaler AM, Ogedegbe G. A senior centerbased pilot trial of the effect of lifestyle intervention on blood pressure in minority elderly people with hypertension. J Am Geriatr Soc 2008; 56: 1860-1866.

25 Piepoli M, Isea JE, Pannarale G, Adamopoulos S, SleigHT P, Coats AJS. Load dependence of changes in forearm and peripheral vascular resistance after acute leg exercise in man. J Physiol 1994; 478: 357-362.

26 Von Känel R. Accumulation of 30 min of moderately intense physical activity is a clinically meaningful treatment to reduce systolic blood pressure in prehypertension. J Hum Hypertens 2008; 22: 444-446.

27 Bennett T, Wilcox RG, MacDonald IA. Post-exercise reduction of blood pressure in hypertensive men is not due to acute impairment of baroreflex function. Clin Sci 1984; 67: 97-103

28 Wilcox RG, Bennett T, Brown AM, Macdonald IA. Is exercise good for high blood pressure? Br Med J 1982; 285: 767-769.

29 Brownley KA, West SG, Hinderliter AL, Ligth KC. Acute aerobic exercise reduces ambulatory blood pressure in borderline hypertensive men and women. Am J Hypertens. 1996; 9: 200-206.

30 Hamer M. The anti-hypertensive effects of exercise: integrating acute and chronic mechanisms. Sports Med 2006; 36: 109-116.

31 Hagberg JM, Montain SJ, Martin III WH. Blood pressure and hemodynamic responses after exercise in older hypertensives. J App/ Physiol 1987; 63: 270-276.

32 Rueckert AP, Slane PR, Lillis DL, Hanson P. Hemodynamic patterns and duration of post-dynamic exercise hypotension in hypertensive humans. Med Sci Sports Exerc 1996; 28: 24-32.

33 Somers VK, Conway J, Coats A, Isea J, Sleight P. Postexercise hypotension is not sustained in normal and hypertensive humans. Hypertens 1991; 18: 211-215.

34 Taylor- Tolbert NS, Dengel DR, Brown MD, McCole SD, Pratley RE, Ferrell RE, Hagberg JM. Ambulatory blood pressure after acute exercise in older men with essential hypertension. Am J Hypertens 2000; 13: 44-51.

35 Mano GMP, Souza VF, Pierin AMG, Lima JC, Ignes EC, Ortega KC, Mion Júnior D. Assessment of the Dixtal DX-2710 automated oscillometric device for blood pressure measurement with the validation protocols of the British Hypertension Society (BHS) and the Association for the Advancement of Medical Instrumentation (AAMI). Arq Bras Cardiol 2002; 79: 601-610.

36 Peixoto-Filho AJ, Mansoor GA, White WB. Effects of actual versus arbitrary awake and sleep times on analyses of 24-h blood pressure. Am J Hypertens 1995; 8: 676-680.

37 Gosse P, Ansoborlo P, Lemetayer P, Clementy J. Daytime and nighttime ambulatory blood pressures should be calculated over the true sleep/waking cycle and not over arbitrary periods. Am J Hypertens 1996; 9: 269-272.

38 Häkkinen K. Neuromuscular fatigue and recovery in women at different ages during heavy resistance loading. Electromyogr Clin Neurophysiol 1995; 35: 403-413.

39 Pingel J, Moerch L, Kjaer M, Langberg $\mathrm{H}$. The influence of training status on the drop in muscle strength after acute exercise. Eur J Appl Physiol 2009; 106: 605-611. 